\title{
Influence of Inter-row Gap Value on Turbine Losses
}

\author{
PAUL G. A. CIZMAS ${ }^{\mathrm{a}, *}$, CORBETT R. HOENNINGER ${ }^{\mathrm{a}}$, SHUN CHEN ${ }^{\mathrm{b}}$ and HARRY F. MARTIN \\ ${ }^{a}$ Department of Aerospace Engineering, Texas A \& M University, College Station, Texas 77843-3141, USA; \\ ${ }^{\mathrm{b}}$ Siemens Westinghouse Power Corporation, Orlando, Florida 32826-2399, USA
}

(Received in final form 23 May 2000)

\begin{abstract}
This paper presents the results of a numerical investigation of the gap influence on the turbine efficiency. The rotor-stator interaction in a 1(1/2)-stage turbine is simulated by solving the quasi-three-dimensional unsteady Euler/Navier-Stokes equations using a parallelized numerical algorithm. The reduced turnaround time and cost/MFLOP of the parallel code was crucial to complete the numerous run cases presented in this paper. The inter-row gap effect is evaluated for 4 gaps, 3 radial positions and 3 angular velocities. As expected, the results presented in this paper show that the efficiency increases and losses decrease while the gap size increases. The maximum efficiency location, however, corresponds to values of the gap size which may be too large for practical use (approximately 1 inch). Fortunately, a local maximum efficiency and minimum losses location has been found at approximately 0.5 inches gap size. The efficiency variation near the local optimum is large, in some configurations being as high as 1.4 points for a gap size variation of only 0.076 inches. Data produced by the numerical simulations can be used to develop a design rule based on the inter-row gap size.
\end{abstract}

Keywords: Unsteady flow; Rotor-stator interaction; Turbine flow; Numerical simulation; Parallel computation

\section{INTRODUCTION}

Numerical simulation of unsteady effects in turbomachinery is a necessary step for advanced design and analysis. The requirement to further increase performance and improve reliability in turbomachinery has motivated designers to better understand unsteady effects. One reason to simulate unsteady flows in turbomachines is to be able to predict dangerous phenomena such as stall flutter or rotating stall. Neither stall flutter nor rotating stall can be predicted using steady flow simulation. These dangerous phenomena might happen due to the fact that blade loading and turbine inlet temperature are constantly increased, quite often pushing the engine out of the envelope

*Corresponding author. Tel.: 979 845-5952, Fax: 979 845-6051, e-mail: cizmas@aero.tamu.edu 
of traditional designs. Another reason to simulate unsteady flows in turbomachinery is to be able to predict rotor-stator interaction. Experimental investigations and numerical simulations have shown that efficiency can be improved by optimizing the circumferential relative position of consecutive airfoil rows. The purpose of this project is to numerically investigate another important parameter of rotor-stator interaction, the interrow gap size. This paper will present the influence of gap size on the turbine efficiency.

An important part of the unsteady effects in turbomachinery results from the rotor-stator interaction. The main sources of unsteadiness present in the rotor-stator interaction are potential flow interaction and wake interaction. Potential flow interaction is a purely inviscid interaction due to the pressure variation caused by the relative movement of the blades and vanes. Potential flow interaction mainly affects adjacent airfoil rows. Wake interaction is the unsteadiness generated by the vortical and entropic wakes shed by one or more upstream rows. These wakes interact with the downstream airfoils and other wakes. Additional sources of unsteadiness present 'in the rotor-stator interaction include vortex shedding, hot streak interaction, shock/boundary layer interaction, and flutter.

One way of taking advantage of the rotorstator interaction effects is through airfoil "clocking" or "indexing". Stator clocking consists of varying the circumferential relative position of consecutive stator airfoils. Consecutive rotor airfoils can be clocked as well, as shown in a previous paper (Cizmas and Dorney, 1999b). The effects of airfoil clocking on turbine performance have been investigated both experimentally and numerically. The experimental results of Huber et al. (Huber, Johnson, Sharma, Staubach and Gaddis, 1996) showed a $0.8 \%$ efficiency variation due to clocking. For the same turbine, a twodimensional numerical analysis for the midspan geometry by Griffin et al. (Griffin, Huber and Sharma, 1996) correctly predicted the maximum efficiency clocking positions. However, the predicted efficiency variation was only $0.5 \%$. Clocking effects in a 1(1/2)-stage turbine have also been numerically simulated by Eulitz et al. (Eulitz, Engel and Gebbing, 1996) and Dorney and Sharma (Dorney and Sharma, 1996). The effects of airfoil clocking on a six-row turbine have been also investigated (Cizmas and Dorney, 1999b). This paper presented for the first time rotor rows clocking, clocking multiple rows and introduced the concept of "fully clocking", i.e., clocking all the rotor and stator rows. In all these analyses, the highest efficiencies occurred when the wake of the upstream airfoil impinged on the leading edge of the downstream airfoil, while the lowest efficiencies were observed when the upstream wake was convected through the middle of the downstream airfoil passage. For the turbine investigated, the clocking of the second-stage produced larger efficiency variations than the clocking of the third-stage. This conclusion was true for both rotor and stator clocking. The predicted results also showed that rotor clocking produces an efficiency variation which is approximately twice the efficiency variation produced by stator clocking.

Another way of taking advantage of the rotorstator interaction is through variation of the interrow gap. If one assumes that the potential flow interaction is not a function of the inter-row gap, then a linear relation could be obtained between the clocking position and the inter-row gap that are producing the same efficiency. However, the potential flow interaction varies with the inter-row gap. Consequently, the relationship between the clocking position and the inter-row gap is more complicated. The focus of the present paper is to investigate the influence of inter-row gap on the turbine efficiency. Once the variation of efficiency with inter-row gap is obtained, a correlation between the clocking position and inter-row gap is possible.

The first part of this paper briefly presents the flow model and the numerical approach. The next part of the paper presents the results of the interrow gap variation on losses and turbine efficiency. 
The paper ends with conclusions and suggestions for future work.

\section{NUMERICAL MODEL}

The PaRSI2 code used to simulate the flow in the turbine is presented in detail in (Cizmas and Subramanya, 1997). The numerical approach used in the code is based on the work done by Rai (Rai and Chakravarthy, 1986). The PaRSI2 code was developed as a parallel version of the STAGE-2 analysis, which was originally developed at NASA Ames Research Center. The numerical approach is briefly described here.

\section{Governing Equations}

The quasi-three-dimensional, unsteady, compressible flow through a multi-stage axial turbomachine with arbitrary blade counts is modeled by using the Navier-Stokes and Euler equations. The computational domain associated with each airfoil is divided into an inner region, near the airfoil, and an outer region, away from the airfoil. The thinlayer Navier-Stokes equations are solved in the regions near the airfoil, where viscous effects are strong. Euler equations are solved in the outer region, where the viscous effects are weak. The flow is assumed to be fully turbulent. The eddy viscosity is computed using the Baldwin-Lomax model and the kinematic viscosity is computed using Sutherland's law.

The Navier-Stokes and Euler equations are written in the strong conservation form. The fully implicit, finite-difference approximation is solved iteratively at each time level, using an approximate factorization method. Two Newton-Raphson sub-iterations are used to reduce the linearization and factorization errors at each time step. The convective terms are evaluated using a third-order accurate upwind - biased Roe scheme. The viscous terms are evaluated using second-order accurate central differences and the scheme is second-order accurate in time. The code has been parallelized using Message Passing Interface libraries and exhibits good parallel efficiency (Cizmas and Subramanya, 1997).

\section{Grid Generation}

The domain is discretized using two types of grids. $O$-grids are used to resolve the Navier-Stokes equations near the airfoil, where the viscous effects are important. The $O$-grids are body-fitted to the surfaces of the airfoils and consequently they provide a good mesh in the critical zones around the leading and trailing edges. Algebraically generated $H$-grids are used to discretize the rest of the passage, permitting the application of periodic boundary conditions without interpolation. The $H$-grids discretize the areas away from the airfoil, where the flow is modeled by the Euler equations. The $O$-grid $/ H$-grid pairs around the airfoil are overlaid and the $H$-grids are patched at the sliding boundary between each stationary and moving blade row. The flow variables are communicated between the $O$ - and $H$-grids through bilinear interpolation.

\section{Boundary Conditions}

Since multiple grids are used to discretize the Navier-Stokes and Euler equations, two classes of boundary conditions must be enforced on the grid boundaries: natural boundary conditions and zonal boundary conditions. The natural boundaries include inlet, outlet, periodic and the airfoil surfaces. The zonal boundaries include the patched and overlaid boundaries.

The inlet boundary conditions include the specification of flow angle, average total pressure and downstream propagating Riemann invariant. The upstream propagating Riemann invariant is extrapolated from the interior of the domain. At the outlet, the average static pressure is specified, while the downstream propagating Riemann invariant, circumferential velocity, and entropy are extrapolated from the interior of the domain. Periodicity is enforced by matching flow 
conditions between the lower surface of the lowest $H$-grid of a row and the upper surface of the top most $H$-grid of the same row. At the airfoil surface, no-slip, adiabatic wall and zero pressure gradient condition are enforced.

For the zonal boundary conditions of the overlaid boundaries, data is transferred from the $H$-grid to the $O$-grid along the $O$-grid's outermost grid line. Data is then transferred back to the $\mathrm{H}$ grid along its inner boundary. At the end of each iteration, an explicit, corrective, interpolation procedure is performed. The patch boundaries are treated similarly, using linear interpolation to update data between adjoining grids (Rai, 1985).

\section{Parallel Computation}

The parallel code uses message-passing interface (MPI) libraries and runs on symmetric multiprocessors (Silicon Graphics Challenge) and massively parallel processors (Cray T3D and Cray T3E). The development of the quasi-three-dimensional parallel code was done such that a threedimensional parallel version can be an easy extension. As a result of this requirement, one processor was allocated for each airfoil in the two-dimensional simulation. Consequently, the number of processors necessary for a typical three-dimensional turbomachinery configuration will not exceed the number of processors available on today's computers. Further details on the parallel algorithm are presented in (Cizmas and Subramanya, 1997).

\section{RESULTS}

\section{Geometry and Flow Conditions}

The results focus on the first three rows of a threestage test turbine. The analysis of the flow in the whole turbine was presented in (Cizmas and Dornery, 1999b; Cizmas and Dornery, 1999a). The first three rows of the test turbine have 58 first-stage stators, 46 first-stage rotors and 52 second-stage stators. A dimensionally accurate simulation of this geometry would require modeling 29 first-stage stators, 23 first-stage rotors and 26 second-stage stators. To reduce the computational effort, it was assumed that there were an equal number of blades (58) in each turbine row. To maintain the same flow area, the airfoils were re-scaled by the factors shown in Table I.

The inlet Mach number is 0.083 and the ratio of the exit static pressure and inlet stagnation pressure is 0.6636 . The ratio of specific heats at the given temperature and pressure is $\gamma=1.284$. The inlet Reynolds number is $1,969,200$ per inch, based on the axial chord of the first-stage stator. The inlet flow angle is 0 degrees and is kept constant for all rotational speeds. The Prandtl number is 1.03 and the turbulent Prandtl number is 1.2875 . Results are presented at hub, mid-span and tip for rotational speeds of 4800, 3600 and 5200 RPM. The hub, mid-span and tip radii are $11,12.25$ and 13.5 inches, respectively. The values of the flow coefficient, $\phi$, used for the numerical simulation are presented in Table II.

To study the variation of losses and efficiency as a function of the inter-row gap size, the size of the inter-stage gap, i.e., the gap between the first-stage rotor and the second-stage vane was varied. The gap between the first-stage vane and the first-stage rotor was kept constant. Results corresponding to four inter-stage gap values are presented in this paper. The four gap values are $0.375,0.518,0.5919$ and 0.883 inches. The length of the axial chord of the first-stage stator is 1.25 inches.

The results presented in this paper were computed using two Newton sub-iterations per time-step and 3000 time-steps per cycle. Here, a cycle is defined as the time required for a rotor to travel a distance equal to the pitch length at

TABLE I Airfoil rescaling factors

\begin{tabular}{lc}
\hline Airfoil & Rescaling factor \\
\hline First-stage stator & 1 \\
First-stage rotor & $46 / 58$ \\
Second-stage stator & $52 / 58$ \\
\hline
\end{tabular}


TABLE II Values of flow coefficient

\begin{tabular}{lccc}
\hline & Hub & Mid & Tip \\
\hline 4800 RPM & 0.3688 & 0.3312 & 0.3005 \\
3600 RPM & 0.4918 & 0.4416 & 0.4007 \\
5200 RPM & 0.3405 & 0.3057 & 0.2774 \\
\hline
\end{tabular}

midspan. To ensure time-periodicity, each simulation was run in excess of 25 cycles.

The computations were performed on the Silicon Graphics Origin2000 computers at NASA Ames and Texas A \& M University and on the Cray T3E computer at Pittsburgh Supercomputing Center. The computation can however be done on any parallel computer, including an inexpensive Beowulf type PC cluster. Five processors were used for this analysis, as shown in Figure 1. The computation time for this simulation was $6.24 \times 10^{-6} \mathrm{secs} / \mathrm{grid}$ point/iteration on the Cray T3E and $7.68 \times 10^{-6}$ secs/grid point/iteration on the Silicon Graphics Origin 2000.

\section{Accuracy of Numerical Results}

To validate the accuracy of the numerical results it is necessary to show that the results are independent of the grid which discretizes the computational domain. Three grids were used to asses the grid independence of the solution. The coarse grid has 37 grid points normal to the airfoil and 112 grid points along the airfoil in the $O$-grid, and 67 grid points in the axial direction and 45 grid points in the circumferential direction in the $H$-grid, as shown in Table III. The number of grid points of the medium and fine grids is also presented in Table III. The medium grid is presented in Figure 2, where for clarity every other grid point in each direction is shown.

The distance between the grid points on the airfoil and the next layer of grid points around the airfoil is the same for the coarse, medium and fine grids in order to have the same $y^{+}$ number. The grid was generated such that, for the given flow conditions, the $y^{+}$number is less than 1 .

The flow in the last row includes the influences of all the upwind rows. As a result, if there are differences between the results due to different grid sizes, these differences will be largest in the last row. For this reason the last row of the turbine was used to assess the grid independence of the numerical results.

Before validating the grid independence of the numerical results, one has to verify that the unsteady solution is periodic. Solution periodicity is assessed by comparing the pressure

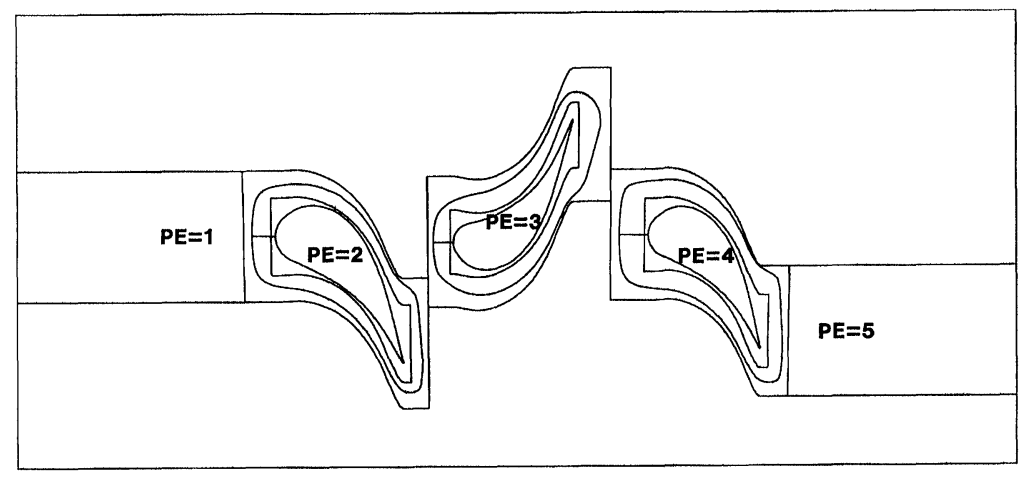

FIGURE 1 Processor allocation. 
TABLE III Grid points of the coarse, medium and fine meshes at mid-span

\begin{tabular}{lcccc}
\hline & $\begin{array}{c}\text { No. of } \\
\text { grids }\end{array}$ & Coarse & Grid points medium & Fine \\
\hline $\begin{array}{l}H \text {-grid inlet } \\
H \text {-grid airfoil, }\end{array}$ & 1 & $75 \times 45$ & $100 \times 60$ & $100 \times 60$ \\
$\begin{array}{l}\text { all rows } \\
\begin{array}{l}O \text {-grid airfoil, } \\
\text { all rows }\end{array}\end{array}$ & 3 & $67 \times 45$ & $90 \times 60$ & $108 \times 72$ \\
$\begin{array}{l}H \text {-grid outlet } \\
\text { Total grid } \\
\text { points }\end{array}$ & 1 & $112 \times 37$ & $150 \times 50$ & $180 \times 60$ \\
\hline
\end{tabular}

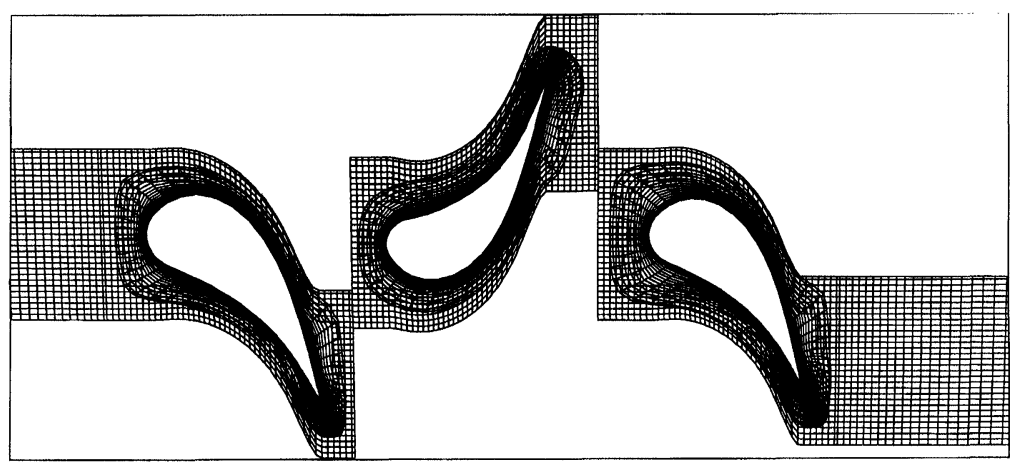

FIGURE 2 Computational grid (every other point in each direction shown).

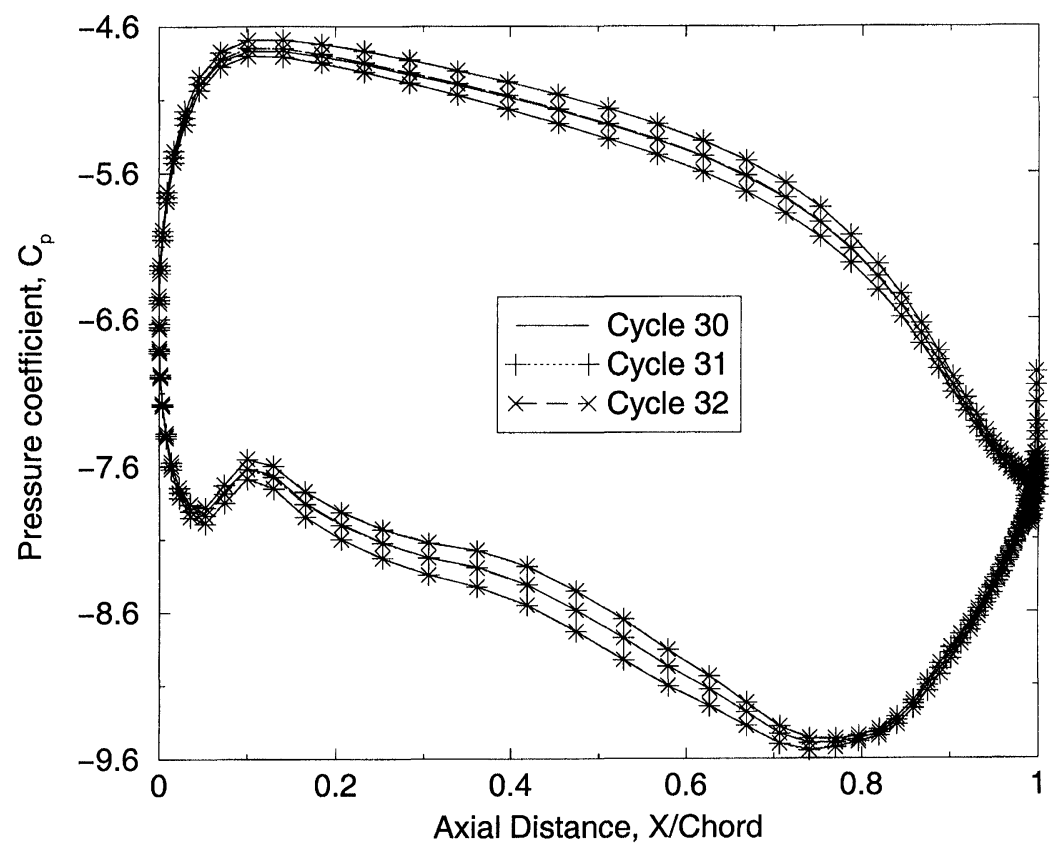

FIGURE 3 Maximum, averaged and minimum pressure coefficients at three consecutive cycles. 


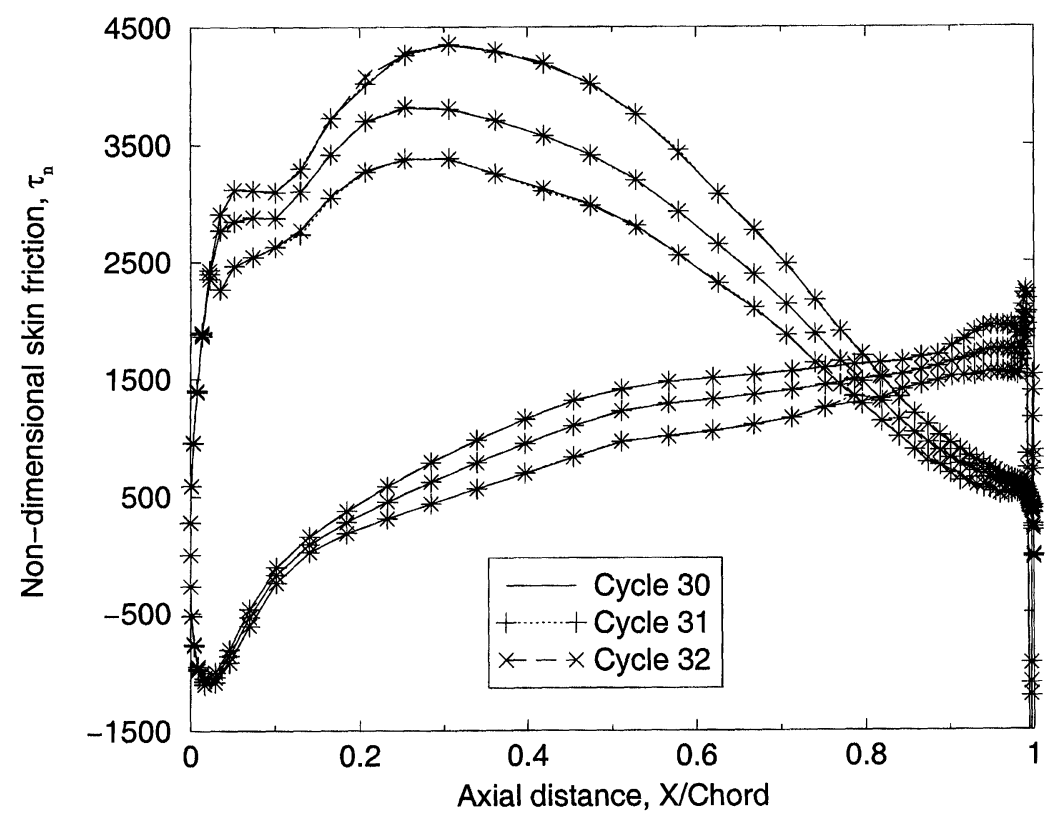

FIGURE 4 Maximum, averaged and minimum skin friction at three consecutive cycles.

coefficient and skin friction results of consecutive cycles, as shown in Figures 3 and 4 . Since the values of the pressure coefficient, $C_{p}$, and skin friction, $\tau$, are almost identical for the three consecutive cycles, one can conclude that the solution is periodic.

Solution periodicity is also proven by the fact that the efficiency averaged over one period reaches an asymptotic value, independent of the cycle number. The variation of the total-to-total efficiency with respect to a fixed baseline efficiency is shown in Figure 5 vs. the number of cycles. The efficiency variation at mid-span radius is shown for the last 20 cycles. In this paper, the flow is considered to be periodic if the variation of averaged efficiency from one cycle to the next one is less than 0.02 points.

To validate the grid independence, three values of the pressure coefficient and skin friction are compared: the averaged, minimum and maximum over one period. The comparison of the pressure coefficients computed using the three grids is presented in Figure 6. Good agreement is obtained among the averaged pressure coefficients of the three grids. Maximum and minimum pressure coefficients corresponding to the medium and fine grids agree well, except around $60 \%$ axial chord on the suction side and $70 \%$ axial chord on the pressure side.

The comparison of the skin friction computed using the three grids is shown in Figure 7. As expected, the skin friction is more sensitive to the grid size variation than the pressure coefficient. Consequently, larger differences among the results corresponding to the three grids are observed compared to the case of the pressure coefficient shown in Figure 6. Even for the averaged values, the skin friction predicted by the coarse grid is significantly different from the skin friction predicted by the medium and fine grids. As shown in Figure 7, the averaged and minimum values of the skin friction predicted by the medium and fine grids agrees rather well. Differences between the maximum skin friction produced by the medium and fine grids are observed at mid-chord, on both suction and pressure sides.

As a result, one can conclude that the medium grid is the best compromise between accuracy 


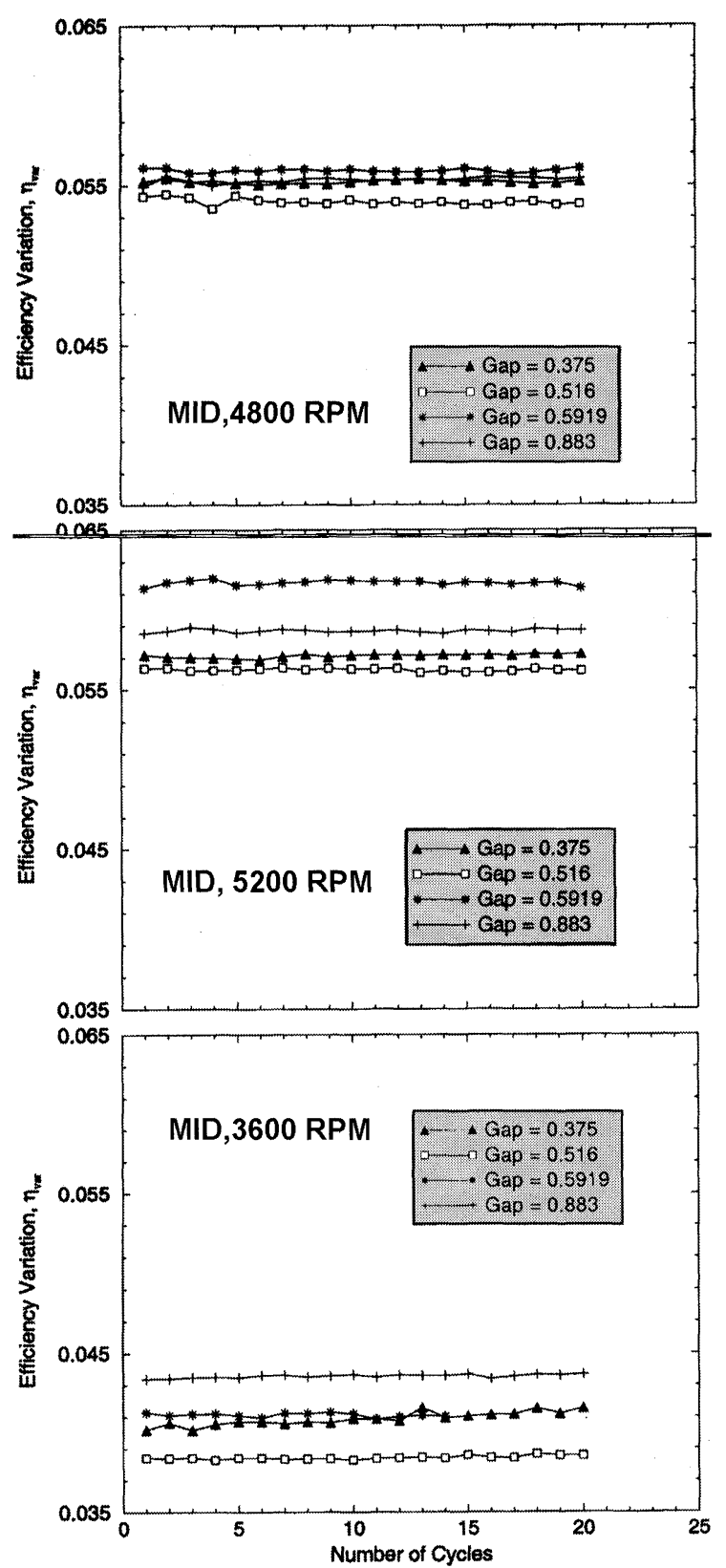

FIGURE 5 Efficiency variation at mid-span vs. number of cycles.

and computational cost. In addition, for the medium and fine grids, the numerical results are independent of the grid size and consequently the numerical accuracy is proven.

\section{Efficiency Variation}

The variation of the total-to-total efficiency as a function of the inter-stage gap, radius and angular velocity is shown in Figure 8. The definition of the total-to-total efficiency is (Lakshminarayana, 1996):

$$
\eta_{t-t}=\left(1-\frac{T_{2, c a, t a}^{*}}{T_{1, c a, t a}^{*}}\right) \frac{1}{1-\left(p_{2, c a, t a}^{*} / p_{1, c a, t a}^{*}\right)^{((\gamma-1) / \gamma)}}
$$

where subscript " $c a$ " denotes circumferentialaveraged, " $t a$ " denotes time-averaged, and the superscript " $*$ " denotes total (or stagnation). To illustrate the efficiency variation as a function of the inter-row gap size, it is sufficient to present the efficiency variation, $\eta_{\mathrm{var}}$, relative to a fixed baseline efficiency, $\eta_{b}$, such that $\eta_{\mathrm{var}}=\eta_{t-t}-\eta_{b}$. The value of baseline efficiency is not specified since it is not critical for the point we are trying to make in this paper. Note that efficiency variation, as opposed to absolute value of efficiency, is shown in Figures 5 and 8.

In Figure 8 the efficiency variation is shown as a function of the inter-stage gap and angular velocity, while the radius is kept constant. For all radial positions, the lowest efficiency corresponds to the low angular velocity (3600 RPM) and the highest efficiency corresponds to the high angular velocity (5200 RPM), except at hub for a gap size value of 0.592 inches. The efficiency increases with the gap value, except for high angular velocity (5200 RPM) at the mid-radius. The efficiency varies significantly around a gap value of approximately 0.5 inches. The efficiency variation can be as high as 1.4 points for a gap size variation of only 0.076 inches.

Besides the maximum efficiency value corresponding to the maximum gap, a local maximum efficiency value exists for intermediate gap values. For tip and hub, the local maximum efficiency corresponds to a gap value of 0.516 inches, for all angular velocities. At mid-radius location, the maximum efficiency at nominal angular velocity and 5200 RPM corresponds to a gap value of 


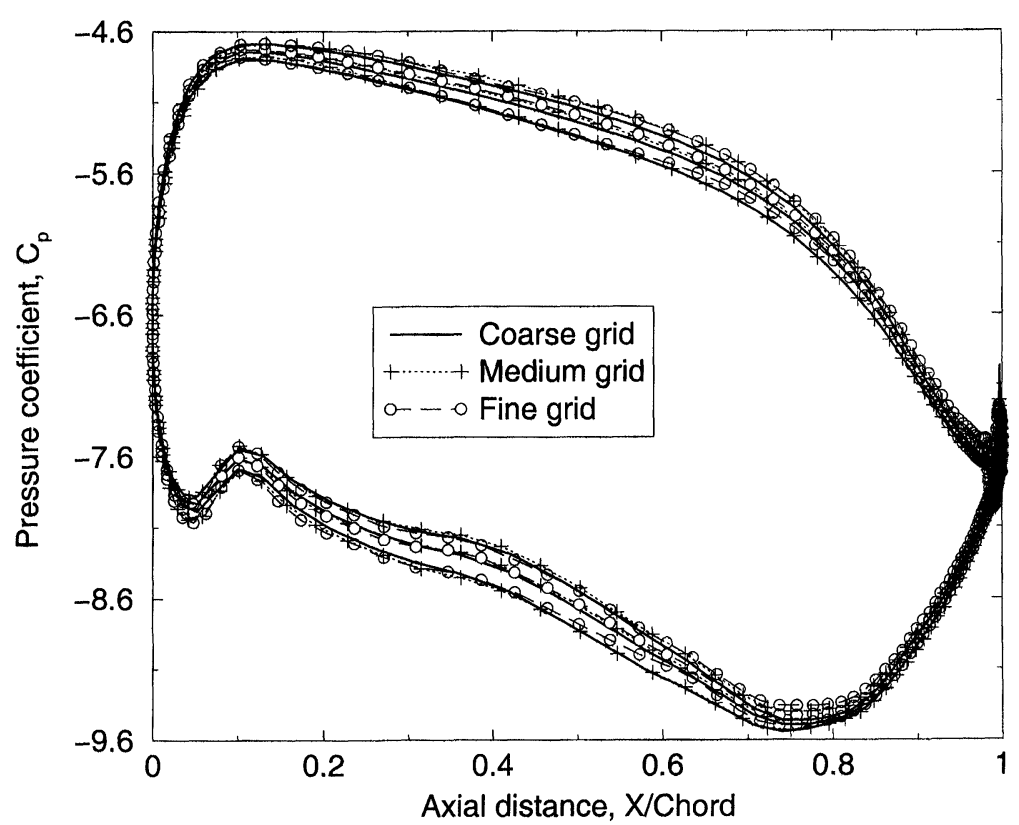

FIGURE 6 Maximum, averaged and minimum pressure coefficient variation on the coarse, medium and fine grids.

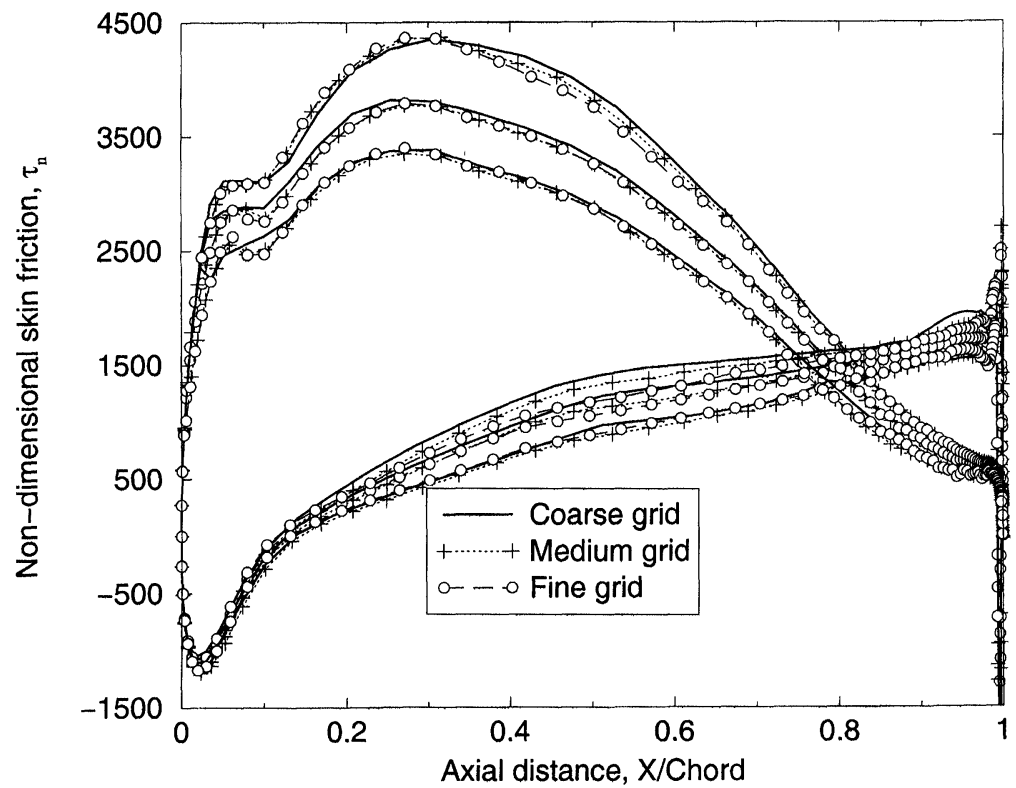

FIGURE 7 Maximum, averaged and minimum skin friction variation on the coarse, medium and fine grids.

0.592 inches. The efficiency variation at 3600 RPM has a minimum value for the 0.516 -inches gap size. For all radial positions, the efficiency variation at nominal angular velocity is less affected by the gap size than at 3600 RPM and 5200 RPM angular velocities. 

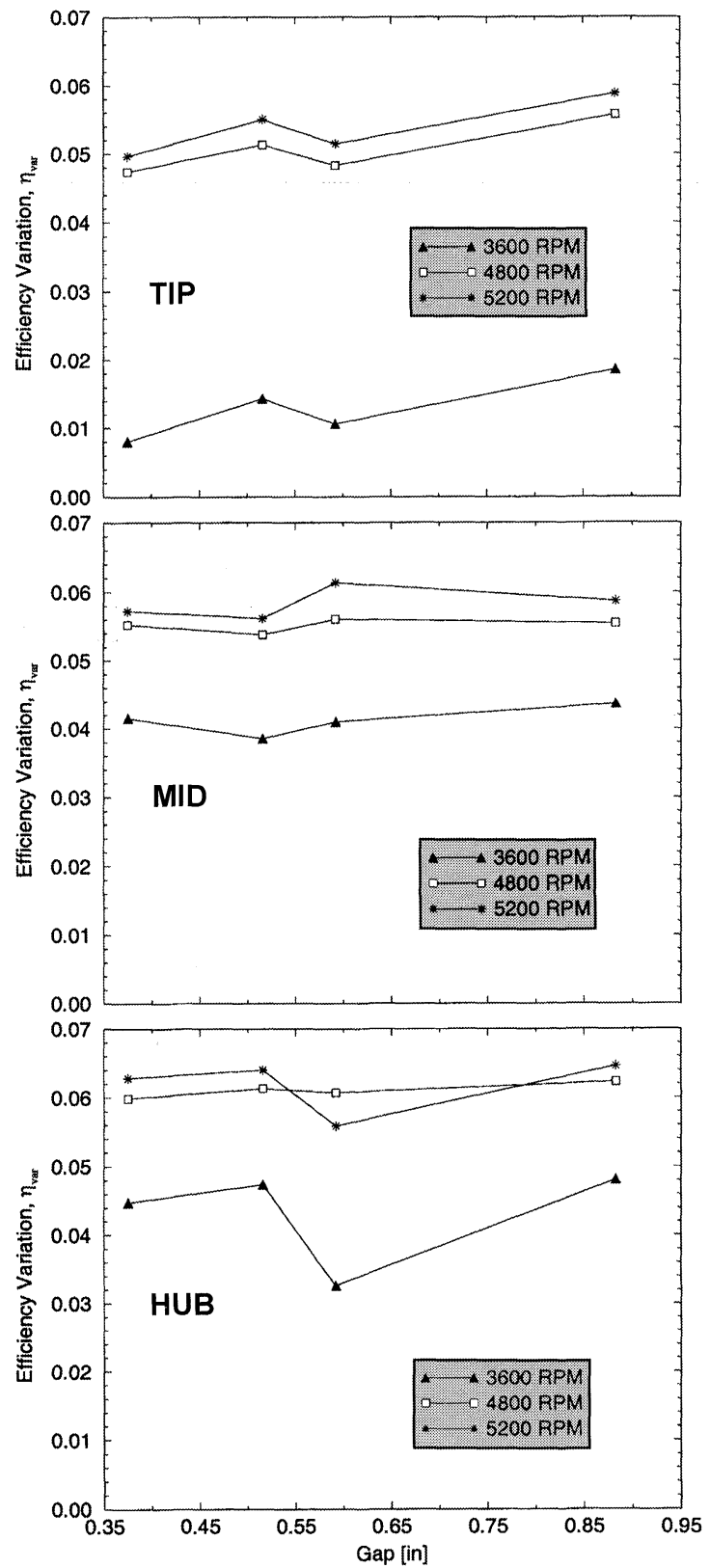

FIGURE 8 Efficiency variation vs. gap at tip, mid-span and hub.

At nominal angular velocity, the largest efficiency corresponds to the hub while the lowest efficiency corresponds to the tip. For the 5200 and 3600 RPM angular velocities, the efficiency at the hub is higher than at the other radii, except for a gap size of 0.592 inches where mid-radius efficiency is the highest. For all angular velocities, the lowest efficiency corresponds to the tip. The lowest tip efficiency corresponds to the 3600 RPM angular velocity. 


\section{Losses Variation}

Turbine losses are presented using the stagnation pressure loss coefficient, $Y$. Similar results can be obtained using the enthalpy loss coefficient, $\zeta_{N}$. The definitions of these coefficients are
(Dixon, 1998):

$$
\begin{gathered}
Y=\frac{p_{1}^{*}-p_{2}^{*}}{p_{2}^{*}-p_{2}} \\
\zeta_{N}=1-\Phi_{N}^{2}
\end{gathered}
$$
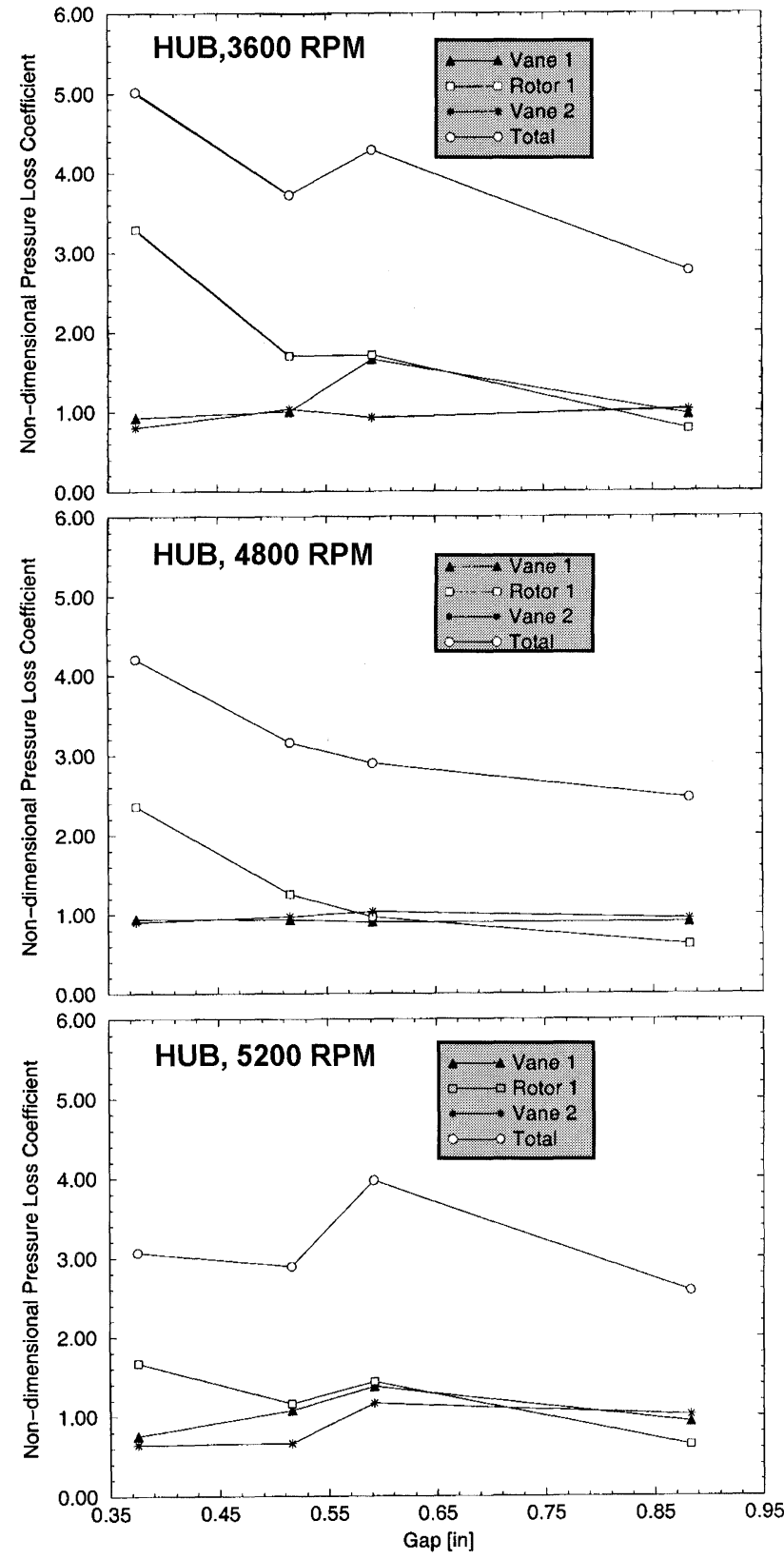

FIGURE 9 Stagnation pressure loss coefficient $Y$ at hub for 3600, 4800 and 5200 RPM. 
where $\Phi_{N}$ is the velocity coefficient, $\Phi_{N}=c_{2} / c_{2 \text { ideal }}$. The total pressures are computed using the absolute velocity in the stator rows and the relative velocity in the rotor row. The losses shown in Figures 9-11 are non-dimensionalized by the value of losses on the first-stage stator at mid-span, gap size 0.518 inches and angular velocity of 4800 RPM.

For low gap values, the rotor $Y$ losses are dominant, as shown in Figures 9-11. Two exceptions are noticed for the $Y$ losses at tip region for nominal angular velocity (4800 RPM)
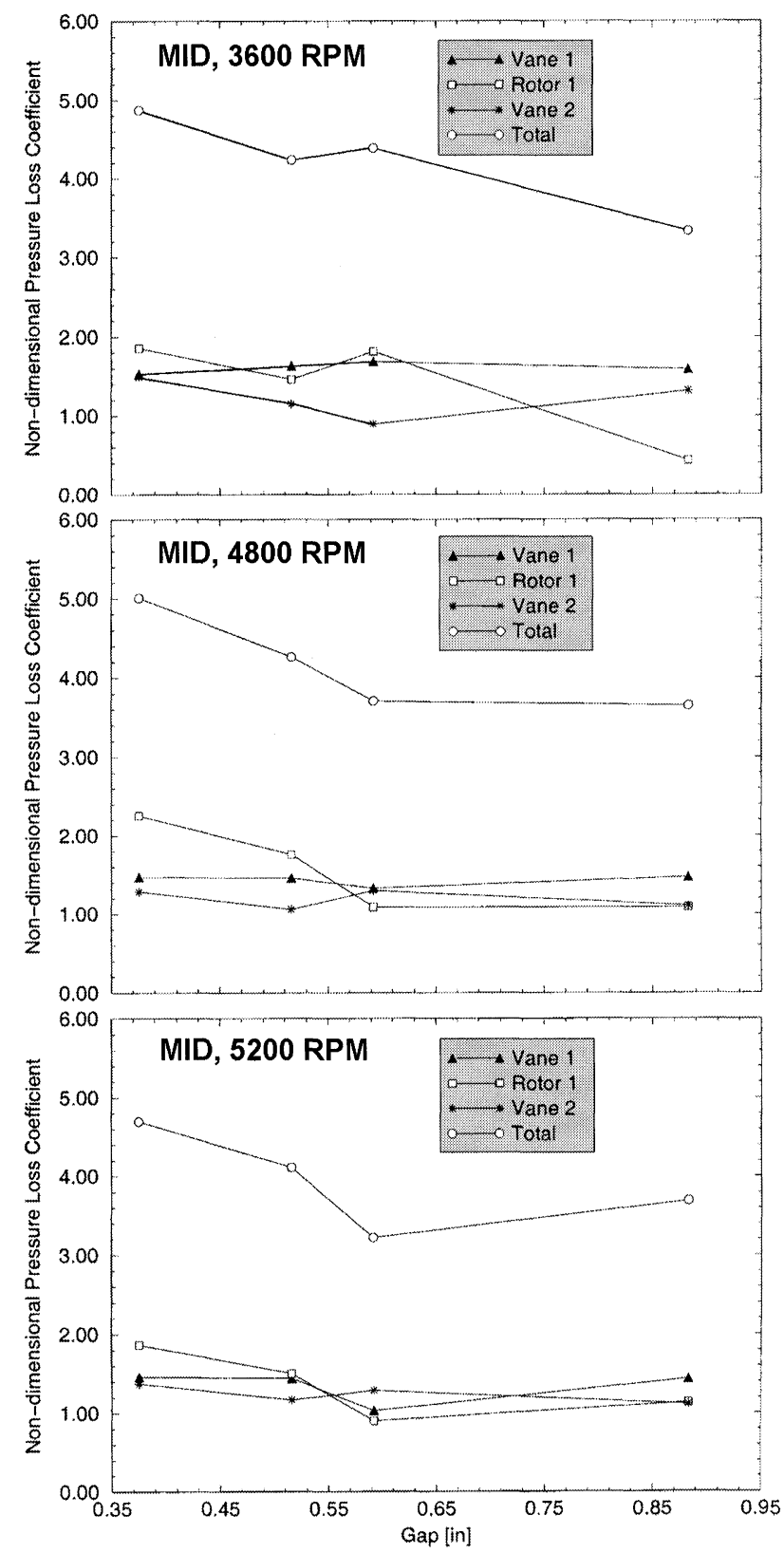

FIGURE 10 Stagnation pressure loss coefficient $Y$ at mid-span for 3600,4800 and 5200 RPM. 

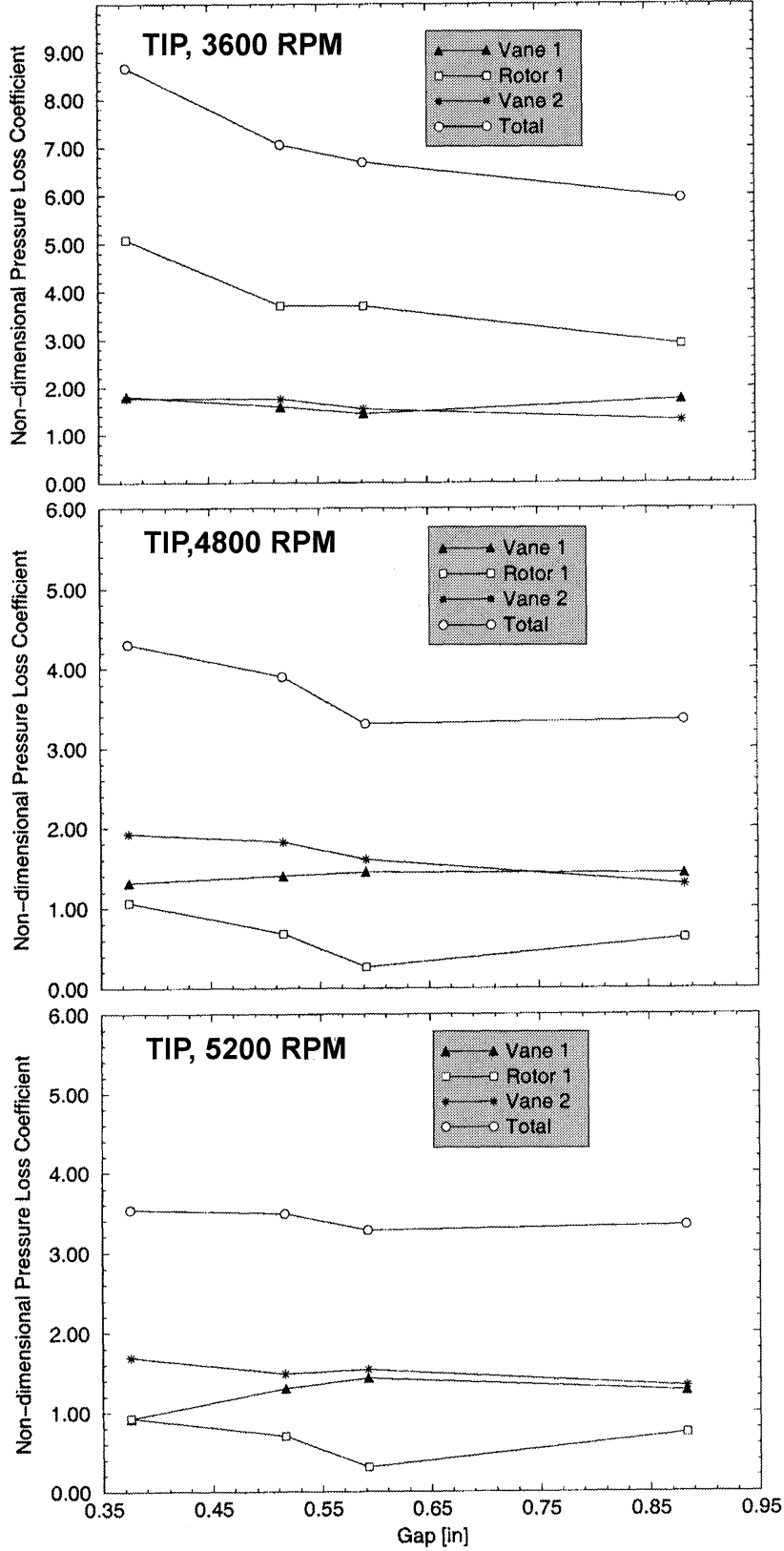

FIGURE 11 Stagnation pressure loss coefficient $Y$ at tip for 3600, 4800 and 5200 RPM.

and at 5200 RPM. In these cases the rotor losses are exceeded by the second-stage vane losses.

The pressure and enthalpy losses most affected by the gap size are the rotor losses. In all the cases, the rotor losses decrease while the gap size increases. As expected, the first-stage vane is least affected by the gap size. The $0.592-$ inches gap produces the minimum losses at midradius for the nominal angular velocity. The same 0.592-inches gap produces the highest efficiency at mid-radius and nominal angular velocity. 


\section{CONCLUSIONS}

A quasi-three-dimensional unsteady Euler/ Navier-Stokes analysis, based on a parallelized numerical algorithm, has been used to investigate the effects of inter-stage gap size in a 1(1/2)stage turbine. Four gap sizes, three angular velocities and three radial locations have been investigated.

The results presented in this paper show that the efficiency increases and losses decrease while the gap size increases. The maximum efficiency and the minimum losses location, however, corresponds to values of the gap size which may be too large for practical use (approximately 1 inch). Fortunately, a local maximum efficiency location has been found at approximately 0.5 inches gap size. Predicting the optimum gap size for local maximum efficiency has a significant impact on turbine aerodynamics, resulting in approximately 0.5 points improved efficiency and 1.5 points reduced losses. Data produced by the numerical simulations can be used to develop a design rule based on the inter-row gap size.

\section{Acknowledgments}

We want to thank Mr. Lynn Layman of Westinghouse Electric Company for making available the resources of the Pittsburgh Supercomputing Center and to Dr. Karen Gundy-Burlet of NASA Ames Research Center for allowing us to access the NASA computers. Last but not the least, we would like to acknowledge the support received from the Texas A \& M Supercomputing Center.

\section{NOMENCLATURE}

$C_{p} \quad$ Pressure coefficient, $C_{p}=\left(p-p_{-\infty}^{*}\right) /[(1 / 2)$ $\left.\rho(\omega r)^{2}\right]$

c Absolute or relative velocity, or chord

$\begin{array}{ll}p & \text { Pressure } \\ r & \text { Radius } \\ T & \text { Temperature } \\ u & \text { Absolute velocity } \\ \eta & \text { Efficiency } \\ \gamma & \text { Ratio of specific heats of a gas } \\ \mu & \text { Viscosity } \\ \omega & \text { Angular velocity } \\ \phi & \text { Flow coefficient, } \phi=u_{-\infty} /(\omega r) \\ \tau_{n} & \text { Non-dimensional skin friction, } \tau_{n}=\mu(\partial u / \\ & \partial y) /\left(\mu_{-\infty}\left(u_{-\infty} / c\right)\right)\end{array}$

\section{Subscripts}

$1 \quad$ Inlet

2 Exit

ca Circumferential-averaged

$t-t$ Total-to-total

ta Time-averaged

$-\infty$ Upstream infinity

\section{Superscripts}

* Total (or stagnation)

\section{References}

Cizmas, P. G. A. and Dorney, D. J. (1999a) The influence of clocking on the unsteady forces of compressor and turbine blades, In: Waltrup, P. J. (Ed.), Fourteenth International Symposium on Air Breathing Engines, Florence, Italy.

Cizmas, P. G. A. and Dorney, D. J. (1999b) Parallel computation of turbine blade clocking, International Journal of Turbo and Jet-Engines, 16(1), 49-60.

Cizmas, P. G. A. and Subramanya, D. J. (1997) Parallel computation of rotor-stator interaction, In: Fransson, T. H. (Ed.), The Eight International Symposium on International Symposium on Unsteady Aerodynamics and Aeroelasticity of Turbomachines, Stockholm, Sweden.

Dixon, S. L. (1998) Fluid Mechanics and Thermodynamics of Turbomachinery, Fourth edn., Butterworth-Heinemann.

Dorney, D. J. and Sharma, O. P. (1996) A study of turbine performance increases through airfoil clocking, 32nd AIAA/ ASME/SAE/ASEE Joint Propulsion Conference, Lake Buena Vista, FL, AIAA Paper 96-2816.

Eulitz, F., Engel, K. and Gebbing, H. (1996) Numerical investigation of the clocking effects in a multistage turbine, International Gas Turbine and Aeroengine Congress, Birmingham, UK, ASME Paper 96-GT-26. 
Griffin, L., Huber, F. and Sharma, O. (1996) Performance improvement through indexing of turbine airfoils: Part IInumerical simulation, ASME Journal of Turbomachinery, 118(3), 636-642.

Huber, F., Johnson, P., Sharma, O., Staubach, J. and Gaddis, S. (1996) Performance improvement through indexing of turbine airfoils: Part I - experimental investigation, $A S M E$ Journal of Turbomachinery, 118(3), 630-635.
Lakshminarayana, B. (1996) Fluid Dynamics and Heat Transfer of Turbomachinery, Wiley, Chapter 2, p. 58.

Rai, M. M. (1985) Navier-Stokes simulation of rotor-stator interaction using patched and overlaid grids, AIAA 7th Computational Fluid Dynamics Conference, Cincinnati, Ohio, AIAA Paper 85-1519.

Rai, M. M. and Chakravarthy, S. (1986) An implicit form for the Osher upwind scheme, AIAA Journal, 24, 735-743. 

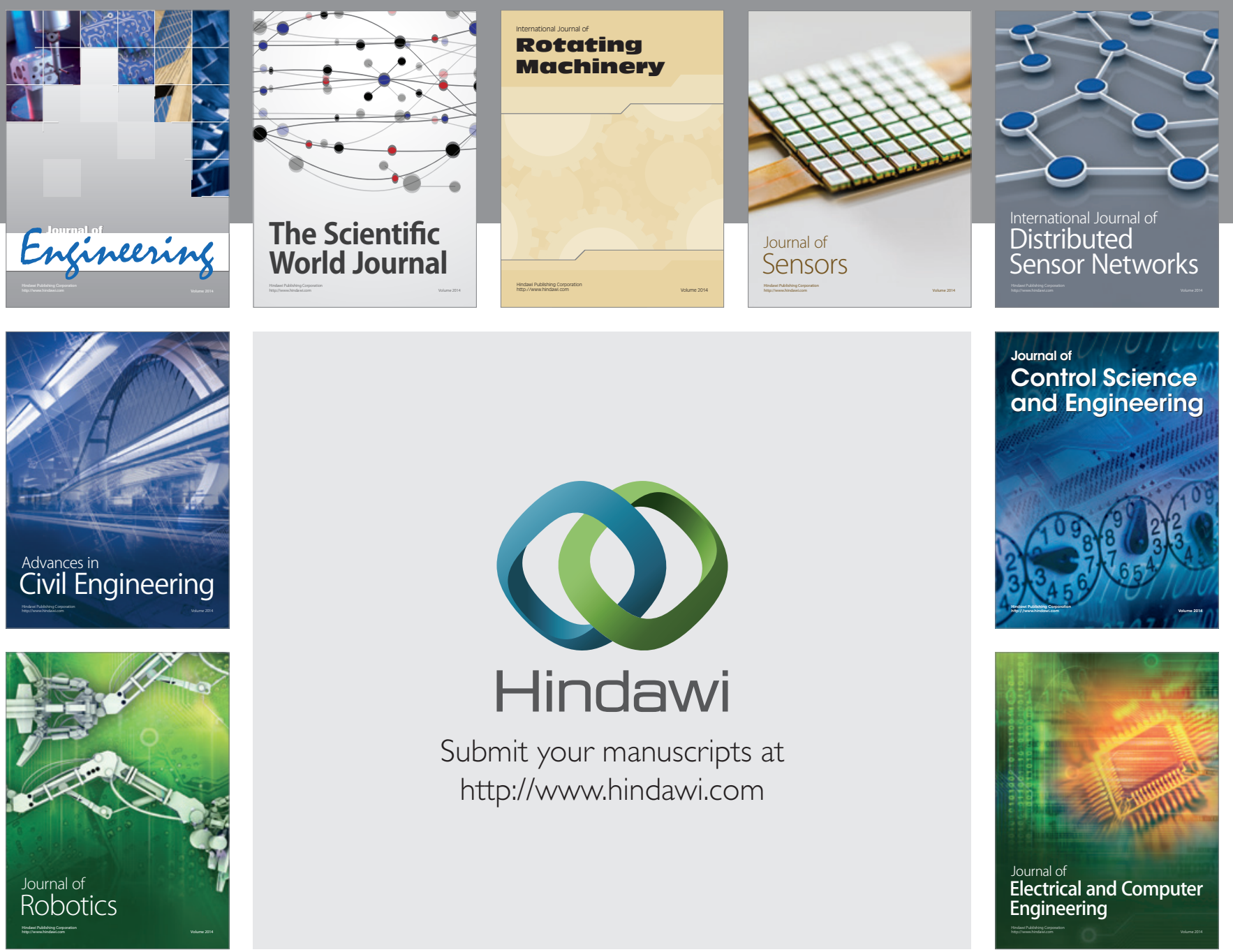

Submit your manuscripts at

http://www.hindawi.com
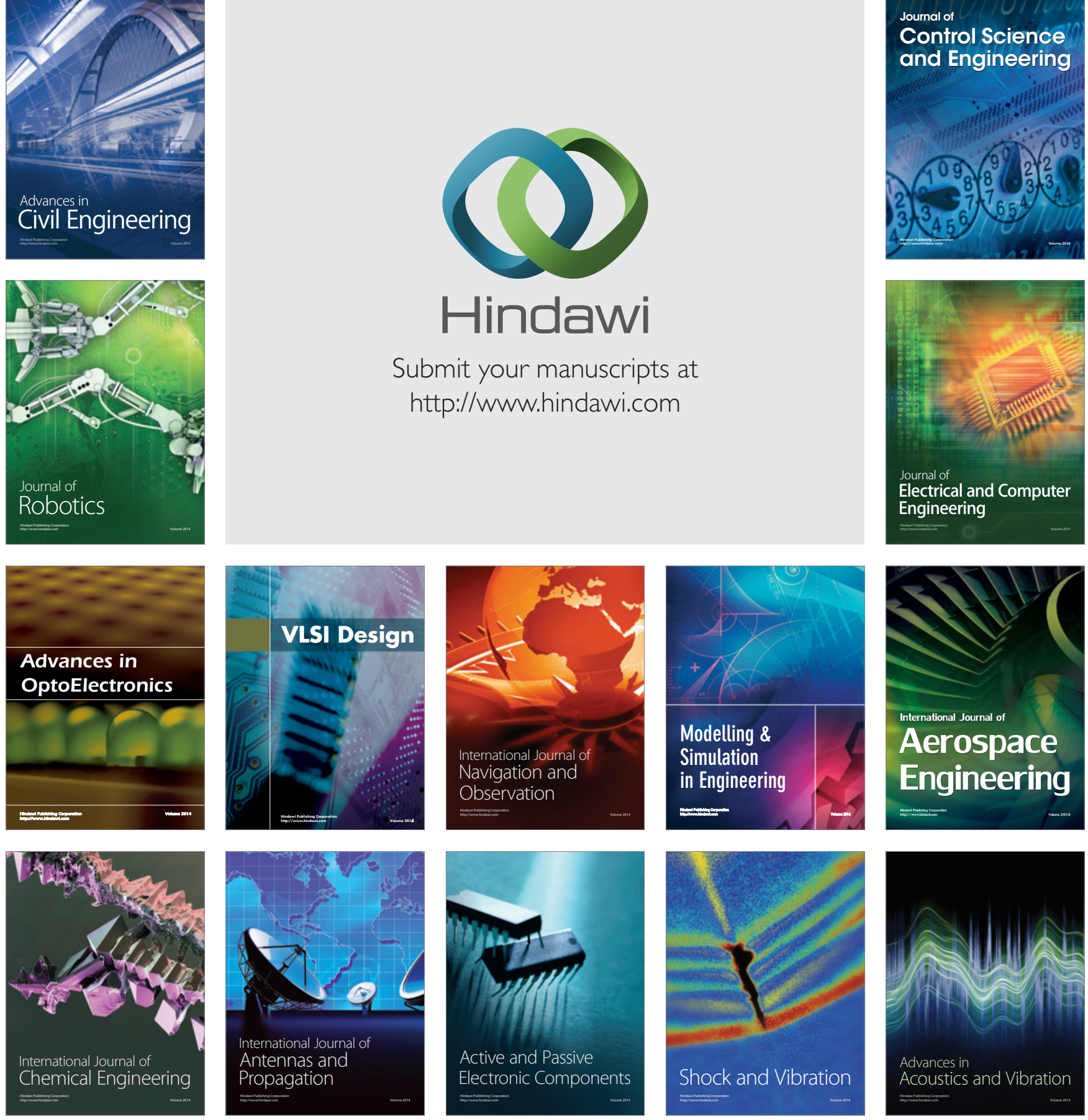